\title{
High-overtone Bulk Acoustic Resonator as passive sensor acting as buried cooperative target interrogated by Ground Penetrating RADAR
}

\author{
J.-M Friedt*, A. Saintenoy ${ }^{\dagger}$, T. Baron ${ }^{\ddagger}$, É. Lebrasseur ${ }^{\ddagger}$, T. Laroche $^{\ddagger}$, S. Ballandras ${ }^{\ddagger}$, M. Griselin ${ }^{\S}$ \\ ${ }^{*}$ SENSeOR, Besançon, France, Email: jmfriedt@femto-st.fr \\ †IDES, UMR CNRS 8148, Université Paris Sud, France \\ $\ddagger$ FEMTO-ST Time \& Frequency department, UMR CNRS 6174, Besançon, France \\ $\S$ ThéMA, UMR CNRS 6049, Université de Franche Comté, Besançon, France
}

\begin{abstract}
We consider a broadband bulk acoustic resonator architecture - the so called High-overtone Bulk Acoustic Resonator (HBAR) - as a transducer acting as cooperative target to a RADAR interrogation unit. Specifically, we consider the compatibility of such an acoustic device as a passive buried sensor interrogated through a wireless link by an unmodified GroundPenetrating RADAR (GPR). While the comb of modes is detected as a series of echoes well within the typical interrogation duration of GPRs, with a spacing between adjacent echoes representative of the acoustic velocity and hence the physical quantity under investigation, the poor coupling coefficient of each individual mode due to the spreading of the piezoelectric transducer coefficient over many modes reduces the interrogation range with respect to the acoustic delay line approach. A sensor identification scheme within the clutter of reflections from dielectric buried interfaces is proposed.
\end{abstract}

\section{INTRODUCTION}

The High-overtone Bulk Acoustic Resonator (HBAR) design, based on a stack of materials [1], was originally considered as a solution for increasing the operating frequency of resonators while keeping a robust mechanical setup and high quality factor while removing the lithography constraint associated with patterning electrodes in a surface acoustic wave approach. Based on a stack of a thin piezoelectric active layer on top of a low-loss thick substrate, the transfer function of such a device is intrinsically broadband and characterized by a comb of modes. The mode spacing is given by the low-loss substrate thickness, while the envelope is given by the transfer function of the thin piezoelectric film and characterized by the overtones which are odd multiples of the fundamental frequency.

Using HBAR transducers as sensors has been considered early after the initial design considerations, whether for sensing physical properties [2], [3] or for gravimetric sensing [4].

\section{HBAR AS PASSIVE WIRELESS SENSORS}

The use of an HBAR as a wireless sensor is less common [5] than frequency source applications, and requires different design considerations: rather than focusing on a high quality factor which must only be high enough for the resonator discharge to last longer than the clutter, loading the resonator requires a significant electro-mechanical coupling. Defined as the ratio of the acoustic to electrical energy, a high enough coupling coefficient is needed to load the transducer during a wireless interrogation following a strategy similar to pulsedRADAR probing [6].

Since an HBAR operates by transferring the acoustic energy from the piezoelectric layer to the thick substrate, the coupling coefficient of the piezoelectric substrate is transferred more or less efficiently to the HBAR modes and opens a design consideration for yielding HBAR transducers most suitable for wireless probing. The multiple modes of the HBAR under investigation here (Fig. 1, modes spaced by $4.6 \mathrm{MHz}$ ) distribute the thin film coupling coefficient to all the modes. Even though $163^{\circ}$-lithium niobate exhibits a strong coupling of up to $K^{2}=50 \%$, distributing this value to all the modes yields a low coupling for individual modes, explaining the poor interrogation range [7] with respect to a delay line [8] designed on a strongly coupled material. Interestingly, in the particular case of the device characterized in Fig. 1, the sum of all coupling coefficients defined as $K^{2} \simeq \frac{\pi^{2}}{4} \times \frac{f_{a}-f_{r}}{f_{r}}$ with $f_{r}$ and $f_{a}$ the resonance and anti-resonance respectively [9] reaches $50 \%$, or the material coupling coefficient of the active piezoelectric layer.

We thus consider two contributions to a figure of merit of the suitability of an HBAR as passive wireless sensor. On the one hand the coupling coefficient $K$ defines the efficiency of the electromagnetic to mechanical conversion - or the ratio of the incoming electromagnetic energy to the stored mechanical energy - and should be maximized to improve the returned power level. The interrogation range is directly related to this quantity since in the RADAR equation, the equivalent cross section of the cooperative target is replaced with $K^{2}$ since the wave is converted from electromagnetic to mechanical and back to electromagnetic, multiplied by the internal acoustic losses of the transducer: a drop by a factor of 10 of the coupling coefficient yields an interrogation range drop of 3 since the cross section appears as the fourth-root power in the RADAR equation. On the other hand the quality factor $Q$ defines the ability of the device to store energy through the ratio of the energy lost for each oscillation period to the stored energy. The quality factor of each individual mode should be high enough to clearly separate each mode contribution and thus prevent the comb of modes to blur in a continuous envelope in which individual echoes would no longer be detectable. Hence, the considered figure of merit is 
$K^{2} \times Q$ (no unit). [10]

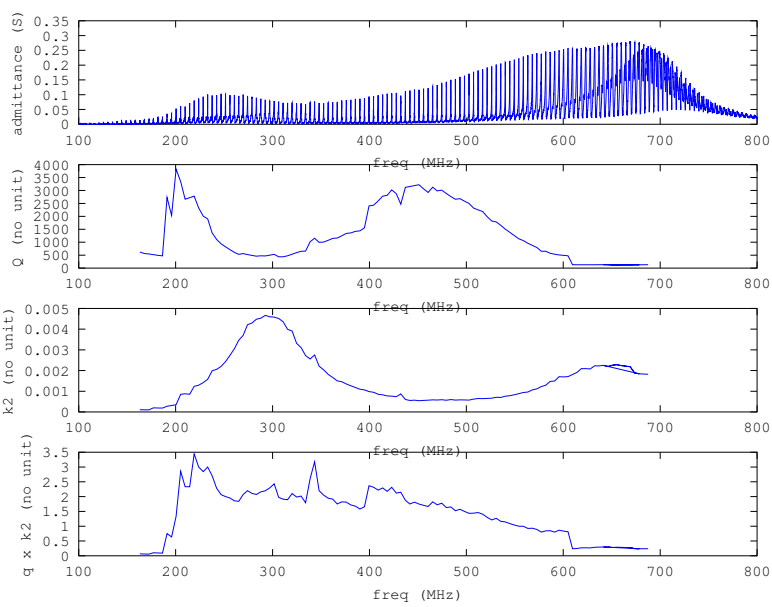

Fig. 1. Top to bottom: experimental admittance of the HBAR (lithium niobate on quartz) used in this report, quality factor and coupling coefficient. A figure of merit $K^{2} \times Q$ is deduced, decreasing with frequency within the frequency range under investigation

This observation yields some consideration as to the design of HBARs for use as passive wireless sensor. High quality factor, as required for frequency source applications, is no longer the most significant design factor: for wireless sensing, the incoming electromagnetic energy must be efficiently converted to a mechanical wave which itself is radiated back to the interrogation hardware. This electro-mechanical conversion coefficient $K$ is unequally distributed over the modes as shown by modelling a lithium niobate $\left(\mathrm{YXl} / 163^{\circ}\right.$-rotated cut) atop corindon (sapphire) stack (Fig. 2. Two cases are investigated: multitude of closely spaced modes when the low-loss substrate is thick $(800 \mu \mathrm{m})$ or a few widely spaced (in the frequency domain) modes due to a thin substrate $(100 \mu \mathrm{m})$. In the latter case, the distribution of the piezoelectric material $K^{2}$ over a few modes yields individual mode electro-mechanical coupling coefficients of a few percents, dropping by a factor of 10 in the case of a dense comb of modes. We hence conclude with a design tradeoff when manufacturing HBAR transducers dedicated to wireless sensing: the mode spacing must be low enough for the time domain echoes (separated by a delay equal to the inverse of the frequency spacing) to be well separated, while being large enough to minimize the number of modes in the piezoelectric layer envelope and hence maximize the coupling coefficient of each individual mode. We observe in both cases that the sum of the individual mode electromechanical coefficients yields a value of $47.5 \% \pm 0.5 \%$, in good agreement with the electro-mechanical coupling coefficient of the piezoelectric layer.

Furthermore, operating within the envelope of the fundamental mode of the thin piezoelectric layer is best suited since the piezoelectric thin film coupling coefficient $K$ of the overtones drops as the overtone number $N$ itself (i.e. $K^{2}$ drops as $N^{2}$, or a 9 to $14 \mathrm{~dB}$ drop when operating on $N=3$ or $N=5$ respectively with respect to the fundamental mode [11]). Hence, rather than operating at high frequency by using an overtone of the thin piezoelectric layer, the HBAR is best designed for operating as a wireless transducer by using a thinner piezoelectric layer so that the investigated modes remain in the fundamental mode of the active layer (Fig. 3).
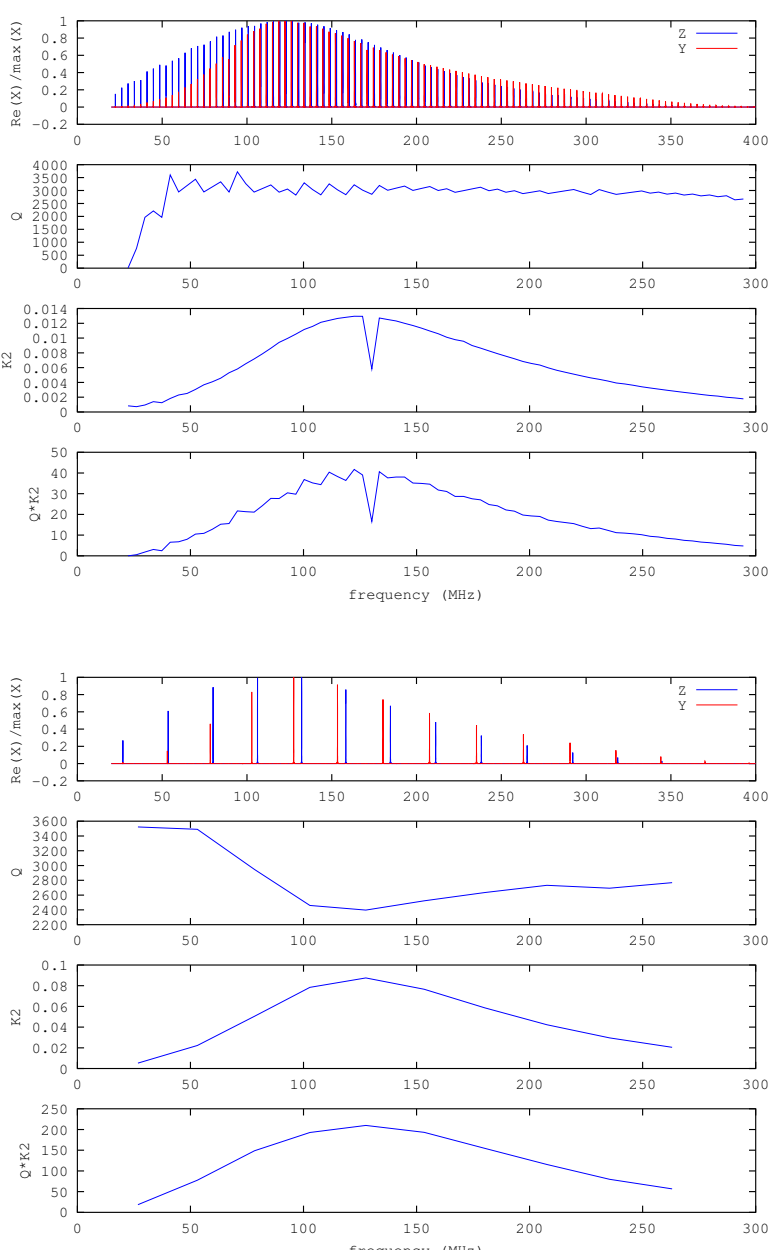

Fig. 2. Simulation of two configurations of HBARs in which a $\mathrm{YX1} / 163^{\circ}$ lithium niobate layer is located atop a corindon substrate either $800 \mu \mathrm{m}$ thick (top) or $100 \mu \mathrm{m}$ thick (bottom). The thin piezoelectric film thickness defines the mode envelope while the substrate thickness defines the mode spacing. Top displays the normalized impedance $(Z$ in blue) and admittance $(Y$ in red), from which the quality factor (width at half height of the real part of the admittance) and the electro-mechanical coefficient $K^{2}$ - proportional to the distance of the admittance to impedance real parts maxima position. Finally, a figure of merit $Q \times K^{2}$ is computed.

Using the simulated data displayed on this figure, we have verified that the sum of the coupling coefficients of the modes within the envelope of the fundamental mode is $49.9 \%$ (close to the expected $50 \%$ of the lithium niobate layer), the sum of the coupling coefficients of the modes lying within the third overtone envelope is $3 \%$, close to $49.9 / 9=5.5 \%$, and the sum of the coupling coefficients of the modes lying under the fifth overtone is $0.8 \%$, close to the expected $49.9 / 25=2 \%$.

\section{GPR FOR PROBING PASSIVE COOPERATIVE TARGETS}

An alternative to the spectral approach of identifying a resonance frequency using a reflective frequency-sweep network analyzer to track the operating frequency of a single mode (FMCW based RADAR strategy) is to operate in the time domain. A HBAR is best suited for a time domain analysis since the Fourier transform of a comb of modes in the frequency domain is a comb of echoes in the time domain. 

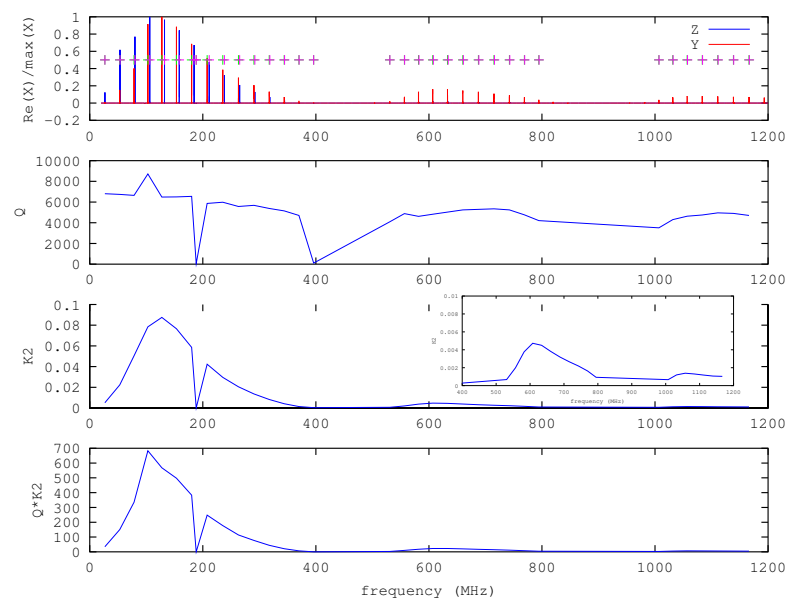

Fig. 3. Simulation of the $S_{11}$ transfer function of an HBAR computed over a frequency range including the fundamental (0-500 MHz), third (500$900 \mathrm{MHz}$ ) and fifth overtone - on the top graph, the crosses indicate the position of the modes identified and automatically processed for computing the quality factor and the coupling coefficient. The fundamental mode of the piezoelectric layer extends from $0-500 \mathrm{MHz}$, the third overtone from 500 to 900 , and the fifth overtone is above $900 \mathrm{MHz}$. The quality factor $Q$ and electromechanical coupling coefficient $K^{2}$ are computed as well as the figure of merit $K^{2} \times Q$, indicating that the only the fundamental mode of the piezoelectric layer should be considered. Inset of the third-from-top graph: zoom on the coupling coefficient of the third and fifth overtone of the piezoelectric layer.

A pulsed mode RADAR emitter as used in Ground Penetrating RADAR (GPR [12]) application is based on unloading a capacitor polarized by a high voltage $(350 \mathrm{~V}$ in a Malå RAMAC unshielded-antenna emitter unit) in an avalanche transistor: the time constant of the resulting pulse is defined by the antenna reflection coefficient $\left(S_{11}\right)$ minimum. This $S_{11}$ minimum is given by the physical antenna dimensions and the surrounding medium permittivity. Practical use of a GPR in an environment ranging from ice to snow and water filled areas induces significant variation in the central frequency of the pulse spectrum (Fig. 4). In the classical patch-antenna equivalent permittivity approach, the GPR dipole antenna of length $d$ operating frequency $f=\frac{c_{0}}{2 d \sqrt{\varepsilon_{r}(e f f)}}$ depends on the ground permittivity $\varepsilon_{r}$ through $\varepsilon_{r}(e f f)=1 / 2 \times\left(\varepsilon_{r}+1\right)$ with $c_{0}$ the electromagnetic velocity in vacuum [13, p.817]. Considering that $\varepsilon_{r} \in[3 . .15]$, the operating range might vary by up to a factor of 2 .

This variable operating frequency is a strong incentive towards the HBAR thanks to the availability of modes in nearly one decade of frequency span. The dependence of these modes with the physical quantity under investigation is still an open question since the distribution of energy in the various materials of the stack is dependent on the overtone index. In order to illustrate this issue, the HBAR presented in Fig. 1 was probed using a GPR fitted with unshielded $200 \mathrm{MHz}$ antenna (best suited for glacier investigation in this case) and then $500 \mathrm{MHz}$ antenna (best suited for civil engineering investigations).

Due to the multitude of modes and hence of the echoes generated by the HBAR, identifying which echo is due to the sensor and which echo is due to passive buried interfaces is mandatory to apply a cross-correlation algorithm and identify the time delay in order to extract the monitored physical quan-
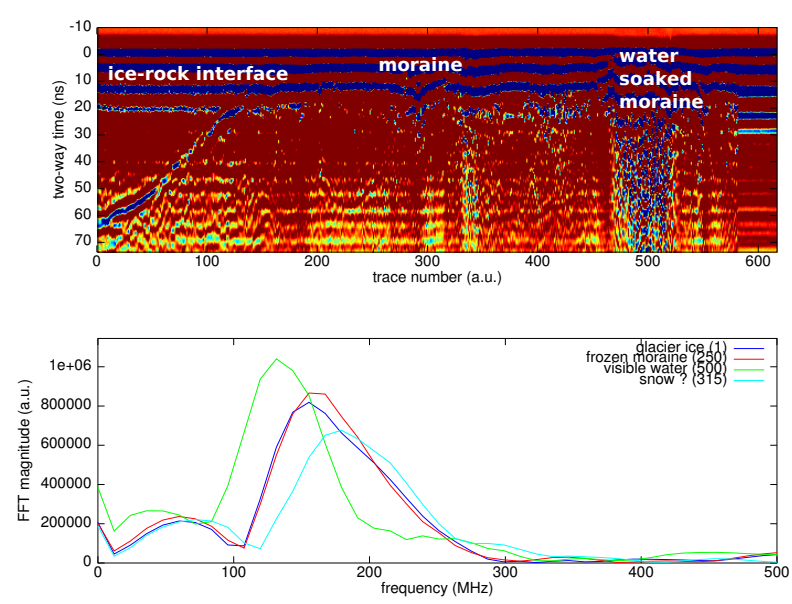

Fig. 4. Top: experimental B-scan GPR measurement over an area ranging from glacier ice (traces 0-100, with a clear interface from the bedrock visible for echoes delayed from 60 to $0 \mathrm{~ns}$ ), to the moraine and surface-soaked water areas around trace 500, yielding the largest permittivity region and hence lowest operating frequency. Bottom: Fourier transform of the emitted pulse depending on the monitored region - the number next to the legend refers to the trace number as observed on the abscissa of the B-scan.
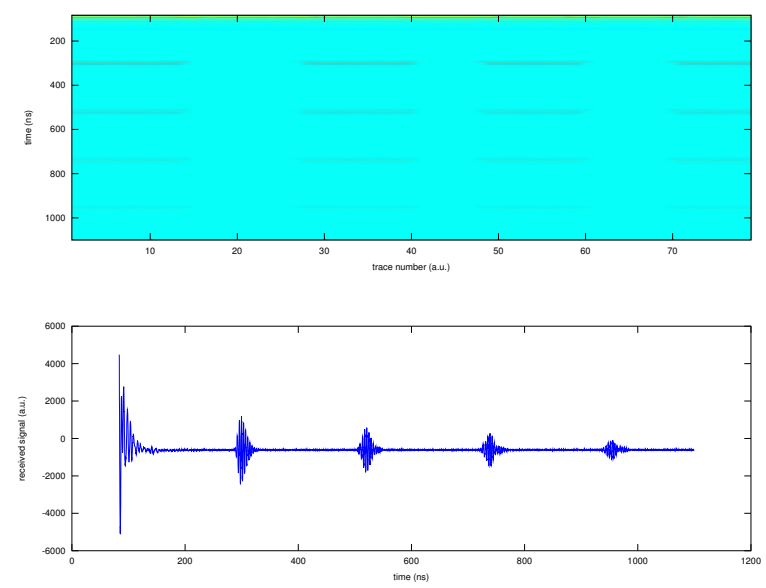

Fig. 5. Top: experimental B-scan of the HBAR sensor probed using a $200 \mathrm{MHz}$ unshielded antenna, with-without-with... sequences of the HBAR located under the GPR. Bottom: experimental A-scan time domain reflections of the HBAR echoes recorded by the GPR.

tity. The classical Kirchhoff migration converts the hyperbolas observed in the (time, position) B-scans when a point-like reflector is detected by a scanning GPR: the hyperbola curvature is solely defined by the medium permittivity and the depth at which the target is located. As seen on Fig. 6, the HBAR delays by the time associated with the propagation duration in the acoustic transducer, hence shifting the hyperbola later in the B-scan. The deep hyperbolas are inconsistent with passive reflectors buried deep underground (in this example multiples of $200 \mathrm{~ns}$ or $26 \mathrm{~m}$ assuming a ground relative permittivity of 5) or would require inconsistent permittivities with respect to the surrounding environments. Hence, the shape of the hyperbola is a unique indicator of a HBAR response. Indeed, since the equation of the reflector depth $t$ as the GPR scans along a line of abscissa $x$ is $c^{2} t^{2}=x^{2}+d^{2}$ when probing a reflector located at depth $d$ in a medium exhibiting an electromagnetic velocity $c$, the curvature of the hyperbolas at position $x=0$ 
(hyperbola summit) is

$$
\left.\frac{\partial^{2} t}{\partial x^{2}}\right|_{x=0}=\frac{2}{c d}=\frac{2 \sqrt{\varepsilon_{r}}}{c_{0} d}
$$

so that erroneously identifying a time-delayed echo of the HBAR would yield an unacceptable estimate of the permittivity, especially so if multiple echoes separated by constant time delays are observed.

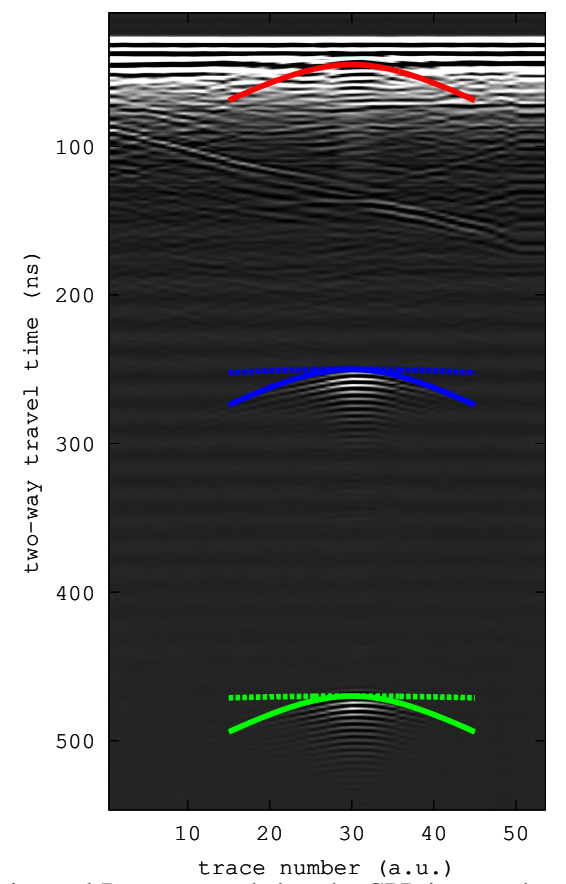

Fig. 6. Experimental B-scan recorded as the GPR is moved over the HBARsensor located $20 \mathrm{~cm}$ in ice. The top-most (red) hyperbola is due to the reflection on the antenna connected to the transducer. Two echoes, delayed by $200 \mathrm{~ns}$ (for a frequency comb spaced by $5 \mathrm{MHz}$ steps), are identified as blue and green hyperbolas with the same shape as the initial reflection. Dashed line: the hyperbola shape if the passive dielectric interface were located at the indicated depth. Solid line: time translated electromagnetic reflection due to the acoustic delay: identifying the sensor reflection from passive interfaces is well defined.

One last unresolved issue is the dependence of the successive echo time delays with the physical quantity under investigation: since the acoustic velocity in the HBAR is a combination of the acoustic velocity in the piezoelectric transducer and the low loss substrate, the observed acoustic velocity depends on the energy distribution in these two layers. The relative energy distribution in each of these layers is dependent on the mode number, so that widely different overtone numbers might exhibit significant different dependence to a given physical effect. Hence, the preliminary calibration between the echo (time) separation and the physical quantity might be no longer valid if the probing frequency significantly differs from the frequency of the calibrated modes, a significant hindrance if the GPR operating frequency varies due to soil moisture content evolution over time and space.

\section{CONClusion}

Having demonstrated the use of High-overtone Bulk Acoustic Resonators as passive cooperative targets suitable for applications in which the sensor is buried and hence no longer accessible for maintenance once installed, probed by a commercially available and unmodified Ground Penetrating RADAR, we describe design rules for optimizing the HBAR architecture to increase the interrogation range while keeping the sensing capability provided by the time delay between successive echoes. Maximizing the coupling coefficient requires operating on the thin piezoelectric film fundamental mode, while the low-acoustic loss substrate thickness is tuned to maximize individual mode coupling while keeping the returned echoes well separated. Identifying the echoes associated with the buried sensor as opposed to passive reflectors is achieved through time-domain separation and hyperbola curvature analysis.

\section{ACKNOWLEDGMENTS}

This research was partly funded by the French National Research Agency (ANR) under the CryoSensors grant, the Université de Franche Comté BQR grant, and supported by the French RENATECH network and its FEMTO-ST technological facility. We acknowledge fruitful discussions with A. Reinhardt (CEA-LETI, Grenoble, France) and E. Lefeuvre (IEF, Orsay, France) concerning the figure of merit of HBAR used as passive sensors.

\section{REFERENCES}

[1] H. Huang, J. Knox, Z. Turski, R. Wargo, and J. Hanak, "Fabrication of submicron $\mathrm{LiNbO}_{3}$ transducers for microwave acoustic (bulk) delay lines," Applied Physics Letters, vol. 24, pp. 109-111, 1974.

[2] S. Alekseev, G. D. Mansfeld, I. Kotelanskii, and V. Veretin, "BAW microwave temperature sensor," in 18th European Frequency and Time Forum (EFTF), 2004, p. 569.

[3] T. Baron, D. Gachon, J.-P. Romand, S. Alzuaga, S. Ballandras, J. Masson, L. Cahterinot, and M. Chatras, "A pressure sensor based on a HBAR micromachined structure," in IEEE International Frequency Control Symposium (IFCS), 2010, pp. 361-364.

[4] G. D. Mansfeld, "Theory of high overtone bulk acoustic wave resonator as a gas sensor," in 13th International Conference on Microwaves, Radar and wireless communications (MIKON), Wrolaw, Poland, 2000, pp. 469-472.

[5] J. Masson, W. Steichen, L. Fagot-Revurat, A. Artieda, P. Muralt, and S. Ballandras, "Hybrid resonant structures for wireless sensor applications," in 23rd European Frequency and Time Forum/IEEE International Frequency Control Symposium, France, 2009, pp. 85-89.

[6] M. Pijolat, D. Mercier, A. Reihardt, E. Defaÿ, C. Deguet, M. Aïd, J. Moulet, B. Ghyselen, and S. Ballandras, "Mode conversion in high overtone bulk acoustic wave resonators," in 22nd European Frequency and Time Forum/IEEE International Frequency Control Symposium, Besançon, France, 2009, pp. 290-294.

[7] J.-M. Friedt, A. Saintenoy, S. Chrétien, T. Baron, E. Lebrasseur, T. Laroche, S. Ballandras, and M. Griselin, "High-overtone bulk acoustic resonator as passive ground penetrating radar coopertive targets," Journal of Applied Physics, vol. 113, no. 13, p. 134904, 2013.

[8] J.-M. Friedt, T. Rétornaz, S. Alzuaga, T. Baron, G. Martin, T. Laroche, S. Ballandras, M. Griselin, and J.-P. Simonnet, "Surface acoustic wave devices as passive buried sensors," Journal of Applied Physics, vol. 109, no. 3, p. 034905, 2011.

[9] S.-Y. Pao, M.-C. Chao, Z. Wang, C.-H. Chiu, K.-C. Lan, Z.-N. Huang, L.-R. Shih, and C.-L. Wang, "Analysis and experiment of HBAR frequency spectra and applications to characterize the piezoelectric thin film and to HBAR design," in IEEE International Frequency Control Symposium and PDA Exhibition, 2002.

[10] V. Kaajakari, "Modeling of MEMS resonators: physics metts appliation requirements," in Joint IEEE International Frequency Control Symposium and European Frequency and Time Forum, San Francisco, USA, 2011, p. tutorial.

[11] J. Vig, "Quartz crystal resonators and oscillators - a tutorial for frequency and timing applications," 2000.

[12] V. Bogorodsky, C. Bentley, and P. Gudmandsen, Radioglaciology. D. Reidel Publishing Company, 1985.

[13] C. Balanis, Antenna Theory, Analysis and Design, 3rd Ed. J. Wiley \& Sons, 2005. 